\title{
Assessment of Nurses' Knowledge and Practice Related to Nursing Care of Children Undergoing Hemodialysis at Assiut City
}

\author{
Abd Alfatah ,A., H ; Ahmad, A., R; Mohamed ,F., Zaky . \\ Department of Pediatric Nursing, Faculty of Nursing, Assiut University \\ Department of Pediatrics,Faculty of Medicine, Assiut university
}

\begin{abstract}
:
Renal failure is a life-threating condition in which there is a buildup of waste and fluid in the body due to severe deterioration of kidney function. The prevalence of both acute and chronic renal failure is high in the Arab World. The aim of this study: is to assess the nurses' knowledge and practice related to nursing care of children undergoing hemodialysis at Assiut city. Descriptive and analytic research design was adopted to meet the aim of this study. Subject and methods: This study was conducted in Pediatric Nephrology and Dialysis Unit in Assuit Children University Hospital, El-Mabarah Hospital and El-Eyman General Hospital. The study included convenience sample of 48 nurses from different Nephrology Units at Assiut City. There are two tools used for collecting data in the study, tool one is a structured interview questionnaire sheet consists of two parts: First Part concerned with personal data of the studied nurses as: snurse' name and age. Second Part concerned with nurses knowledge regarding renal failure and hemodialysis. Tool two a structured observational checklist sheet to evaluate the nurses' practice Result: The main results of study demonstrated that there is no statistical significant difference between the nurses' knowledge and their practice regarding nursing care of children undergoing hemodialysis. There is statistical significant difference between the nurses' knowledge scores and their practice with age. Conclusion: The most of AUH nurses had higher score of knowledge related to renal failure and hemodialysis than the ministry of health hospitals. Nurse's practice regarding hemodialysis children in nephrology unit at Assiut university children hospital is higher than that of nurses working at the Ministry of Heath Hospitals. Recommendations: Continuous nursing educational and in-service training programs on kidney dialysis to upgrade the knowledge and skills of practicing nurses, Periodic monitoring of nurses knowledge and practice to evaluate the level of nurses.
\end{abstract}

Key Words: Renal failure, Hemodialysis, Nurses, knowledge, Practice, Children, Pediatric.

\section{Introduction:}

Renal failure is a life-threatening condition in which there is a buildup of waste and fluid in the body due to severe deterioration of kidney function. The prevalence of both acute and chronic renal failure is high in the Arab World. Data available on the exact prevalence of various renal diseases are very limited. The reported prevalence of chronic renal failure is 225 in Egypt per million population (pmp) and 80 to 120 pmp in the Kingdom of Saudi Arabia. This is in comparison with the reported prevalence of $283 \mathrm{pmp}$ in Europe, 975 pmp in the United States, and 1149 pmp in Japan (Barsoum, 2003).

Renal failure is a condition in which the kidney cannot concentrate urine, conserve electrolytes and excrete waste products. Renal failure in children may occur as an acute or chronic condition. Some cases of acute renal failure resolve without further complication while dialysis is necessary in other children. When acute renal failure continues to progress, it becomes chronic renal failure. Dialysis and kidney transplantation are treatment modalities used for chronic renal failure (Kyle, 2008).

Chronic renal failure is defined as a permanent irreversible destruction of nephron leading to severe deterioration of renal function resulting to end stage renal disease. Causes include congenital kidney and urinary tract abnormalities in children less than 5 years of age and golmerular and hereditary kidney disorders in children 5 to 15 years of age. Signs and symptoms include oliguria, anuria, nocturia, generalized edema, hypertension, nausea, vomiting, numbness and tingling of extremities, muscles twitching, irritability, lethargy, ecchymosis and prolonged bleeding (Datta, 2009) .

Medical management of chronic renal failure includes dialysis to remove waste products and extra water from the blood. There are two types of dialysis; hemodialysis and peritoneal dialysis. Kidney transplantation involves surgically placing a healthy kidney from a donor inside patients' body that is used when there is no life threading medical condition other than kidney failure (Kraut, 2009 and Wolfson, 2009). Hemodialysis is the most common method used to treat advanced and permanent kidney failure. Hemodialysis defined as a medical procedure that uses a special machine to filter waste products from the blood and to restore normal constituents to it ( Perl, 2009).

Nursing responsabilities and role in kidney diseases begins with observation of the child for any 
manifestations that might indicate dysfunction. The most significant ongoing assessment in children with renal conditions are accurate measurements and recording of weight, intake and output and blood pressure. Theses assessment are necessary not only for children with known renal dysfunction but also for those children at risk for developing renal complications.

Pediatric nurse play an important role in management of child undergoing hemodialysis. Before hemodialysis as: providing emotional support to the child, monitoring weight and vital signs, growth measurements, laboratory reports, intake and output, examining the child for signs of infection, observe nutritional status. During hemodialysis as: promote patient comfort, maintain good outflow of blood, monitor for complications as infection and bleeding. And after hemodialysis as: check for any medicine to be given before terminating dialysis, upon removal of fistula needle apply pressure dressing using sterile gause, ask the child to rest at least 15 minutes and dangle his legs to prevent postural hypotension after dialysis, reinforce diet and fluid requirements of child on dialysis, weight the child before leaving the center, remind their about the next schedule of his dialysis (Hockenberry\&Wilson, 2007 and Seppa and Nathan, 2011).

Aim of the study

This study aimed to assess the nurses' knowledge and practice related to nursing care of children undergoing hemodialysis at Assiut city.

\section{Research hypothesis}

- Nurses who has several years of experience are better in knowledge and practice than new graduated nurses.

- Nurses who had training courses are better in knowledge and practice than those who don't have.

\section{Subjects and Method:}

Research design:

Descriptive and analytic research design was used in this study.

\section{Setting:}

The study was conducted in the Pediatric Nephrology and Dailysis Unit. Three hospitals representing Assiut City ; Assiut University Children Hospital, Health Insurance Hospital ( El-Mabarah Hospital ) and El-Eyman General Hospital.

\section{Sampling:}

The study include a convenience sample of 48 nurses from different Nephrology Units at Assiut City (24 nurses from Assiut University Children Hospital, 15 nurses from El-Mabarah Hospital and 9 nurses from El-Eyman General Hospital).
Tools of the study:

\section{Two tools was used in this study}

Tool I: A structured interview questionnaire sheet.It was developed by the researcher and consists of two parts:

First Part: personal data of the studied nurse's as: nurses' names, age, residence, qualifications, marital status, years of experience and their place of work.

Second Part: Questions to assess the nurses' knowledge regarding definition of renal failure, types, causes, signs and symptoms, risk factors, complications and management of chronic renal failure, definition of hemodialysis, complications and nursing management before, during and after dialysis .Knowledge score Poor $<50 \%$, Fair 50-70\%, Good $>70 \%$

Tool II: Nurses practice was assessed and accordingly classified as: vital signs, weight and growth measurement for children undergoing hemodialysis and........ ect.

Nurses' Practice was classified into Inadequate (less than $60 \%$ ) and satisfactory (equal or more than $60 \%$ ). The two tools revised by 5 experts from both nursing and medicine to test its validity.

\section{Method}

\section{Procedure:}

1. Reviewing of the related literature to assess the nurses' knowledge and practice toward renal failure and nursing care of children undergoing hemodialysis.

2. An official approval letter was obtained from the dean of the Faculty of Nursing, Assiut University to ministery of health to the directors of Nephrology Unit at Assiut University Children Hospital, El - Eyman General Hospital and Health Insurance Hospital.

3. Pilot study It was carried out included $10 \%$ of total sample size to evaluate the clarity of the study tools. Accordingly to the modification were done.

4. To conduct this study the researcher took an oral consent from each participant in the different units and every nurse was reassured that information obtained would be confidential and used only for the purpose of the study.

5. The study was conducted over a period of 4 months. It included nurses every other day working at nephrology unit. The studied nurses were interviewed according to their available time hospitals.

6. Interviewing of studied nurses was conducted according to their available time to collect data; two or three nurses were interviewed per day. The time needed for each interview ranged from 20-30 minutes depending upon the individually nurses readiness. 
7. Indirect observation for nursing care given to children undergoing hemodialysis by the researcher was done for each nurse at different hospitals before, during and after hemodialysis using the developed observational checklist. The time taken for observing every nurse ranged from 3-4 hours, only one nurse was observed / day.

8. The study tools were developed by the researcher and the validity of the tool was done by 5 experts in pediatric from both nursing and medicine fields.

\section{Data analysis:}

Data entry and data analysis were done by using SPSS program (statistical package for social science) version 16. Data were presented as number, percentage, mean and standard deviation. Chi square test and fisher exact test were used to compare qualitative data. Pearson correlation was used to measure correlation between score of knowledge and practice. $\mathrm{P}$ value considered statistically significant when $\mathrm{p}<0.05$.

\section{Results:}

Table (1): Distribution of the studied nurses according to their personal characteristics. $(n=48)$

\begin{tabular}{|c|c|c|c|c|c|c|}
\hline \multirow{2}{*}{ Items } & \multicolumn{2}{|c|}{ AUH $(n=24)$} & \multicolumn{2}{|c|}{ MOH $(n=24)$} & \multicolumn{2}{|c|}{ Total $(n=48)$} \\
\hline & No. & $\%$ & No. & $\%$ & No. & $\%$ \\
\hline \multicolumn{7}{|l|}{ Age in years: } \\
\hline$<20$ & 0 & 0.0 & 1 & 4.2 & 1 & 2.1 \\
\hline $20-<30$ & 19 & 79.2 & 13 & 54.2 & 32 & 66.7 \\
\hline $30-<40$ & 5 & 20.8 & 7 & 29.2 & 12 & 25.0 \\
\hline$\geq 40$ & 0 & 0.0 & 3 & 12.5 & 3 & 6.2 \\
\hline Mean \pm SD & \multicolumn{2}{|c|}{$29.17 \pm 3.02$} & \multicolumn{2}{|c|}{$31.38 \pm 6.49$} & \multicolumn{2}{|c|}{$30.27 \pm 5.13$} \\
\hline \multicolumn{7}{|l|}{ Qualification: } \\
\hline Bachelor of Nursing Science & 2 & 8.3 & 1 & 4.2 & 3 & 6.2 \\
\hline Nursing Technical Institute Diplom & 2 & 8.3 & 0 & 0.0 & 2 & 4.2 \\
\hline Nursing Diplom & 18 & 75.0 & 19 & 79.2 & 37 & 77.1 \\
\hline Nursing Diplom + Specialty & 2 & 8.3 & 4 & 16.7 & 6 & 12.5 \\
\hline \multicolumn{7}{|l|}{ Years of experience: } \\
\hline$<5$ & 0 & 0.0 & 6 & 25.0 & 6 & 12.5 \\
\hline $5-10$ & 13 & 54.2 & 7 & 29.2 & 20 & 41.7 \\
\hline$>10$ & 11 & 45.8 & 11 & 45.8 & 22 & 45.8 \\
\hline Mean \pm SD & \multicolumn{2}{|c|}{$9.50 \pm 2.41$} & \multicolumn{2}{|c|}{$9.38 \pm 4.69$} & \multicolumn{2}{|c|}{$9.44 \pm 3.69$} \\
\hline \multicolumn{7}{|l|}{ Attending training courses: } \\
\hline Yes & 0 & 0.0 & 14 & 58.3 & 14 & 29.2 \\
\hline No & 24 & 100.0 & 10 & 41.7 & 34 & 70.8 \\
\hline \multicolumn{7}{|l|}{ Duration of course: } \\
\hline$<3$ days & 0 & 0 & 1 & 7.1 & 1 & 7.1 \\
\hline $3-6$ days & 0 & 0 & 0 & 0.0 & 0 & 0.0 \\
\hline$>6$ days & 0 & 0 & 13 & 92.9 & 13 & 92.9 \\
\hline \multicolumn{7}{|l|}{ Place of training: } \\
\hline In hospital & 0 & 0 & 3 & 21.4 & 3 & 21.4 \\
\hline Out of hospital & 0 & 0 & 11 & 78.6 & 11 & 78.6 \\
\hline
\end{tabular}

Table (2): Distribution of the studied nurses according to their total Level of knowledge about renal failure and hemodialysis at the study setting. $(n=48)$

\begin{tabular}{|l|c|c|c|c|c|c|}
\hline \multirow{2}{*}{\multicolumn{1}{c|}{ Level of knowledge }} & \multicolumn{2}{|c|}{ AUH (n= 24) } & \multicolumn{2}{c|}{ MOH (n= 24) } & \multicolumn{2}{c|}{ Total (n= 48) } \\
\cline { 2 - 7 } & No. & \% & No. & \% & No. & \% \\
\hline Poor & 0 & 0.0 & 0 & 0.0 & 0 & 0.0 \\
\hline Fair & 3 & 12.5 & 5 & 20.8 & 8 & 16.7 \\
\hline Good & 21 & 87.5 & 19 & 79.2 & 40 & 83.3 \\
\hline P-value & \multicolumn{7}{|c|}{$0.701(\mathrm{NS})$} \\
\hline
\end{tabular}


Fisher exact test AUH: Assiut University Hospital * MOH: Ministry of Health Hospitals **

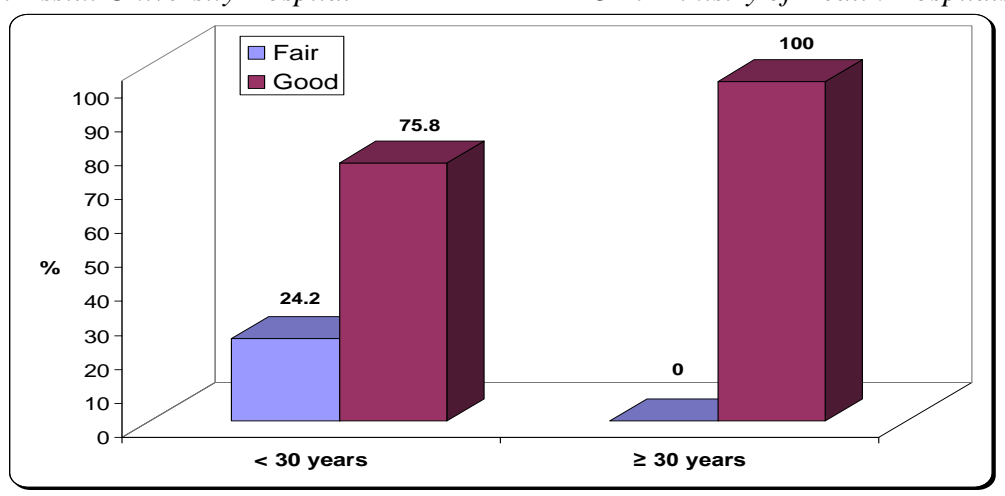

Fig. (1): Relation between total level of nurses' knowledge regarding renal failure, hemodialysis therapy and their age.

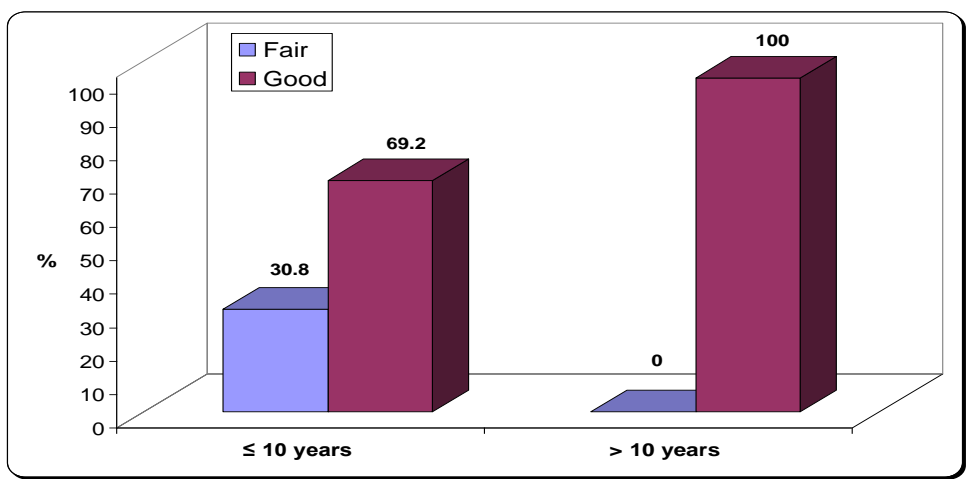

Fig. (2): Relation between total level of nurses' knowledge regarding renal failure, hemodialysis therapy and years of experience.

Table (3): Distribution of the studied nurses according to their total Level of practices about nursing care of children undergoing hemodialysis at the study setting. $(n=48)$

\begin{tabular}{|c|c|c|c|c|c|c|}
\hline \multirow[t]{2}{*}{ Level of practices } & \multicolumn{2}{|c|}{$\begin{array}{r}\mathrm{AUH} \\
(\mathrm{n}=24) \\
\end{array}$} & \multicolumn{2}{|c|}{$\begin{array}{r}\text { MOH } \\
(n=24) \\
\end{array}$} & \multicolumn{2}{|c|}{$\begin{array}{r}\text { Total } \\
(n=48)\end{array}$} \\
\hline & No. & $\%$ & No. & $\%$ & No. & $\%$ \\
\hline Inadequate & 5 & 20.8 & 14 & 58.3 & 19 & 39.6 \\
\hline Adequate & 19 & 79.2 & 10 & 41.7 & 29 & 60.4 \\
\hline P-value & \multicolumn{6}{|c|}{$0.008^{*}$} \\
\hline
\end{tabular}

* Statistical significant difference $(P<0.05)$.

*AUH: Assiut University Hospital.

**MOH: Ministry of Health Hospitals.

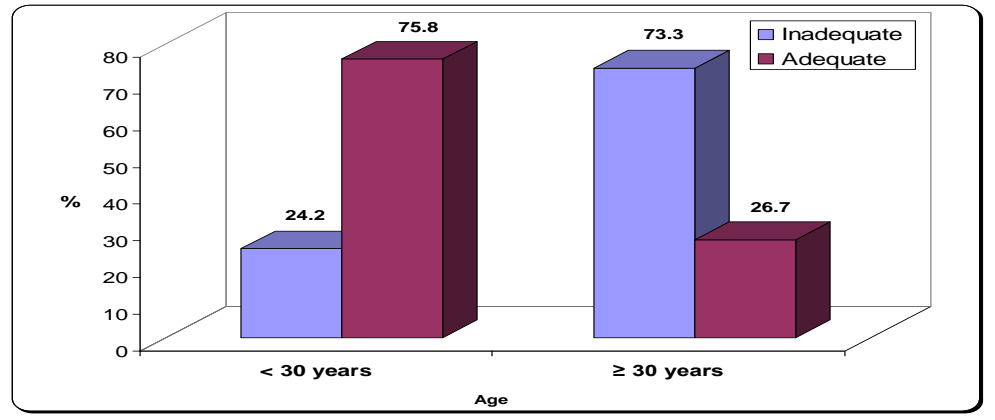


Fig. (3): Relation between total level of practices regarding hemodialysis therapy and age of the studied nurses.

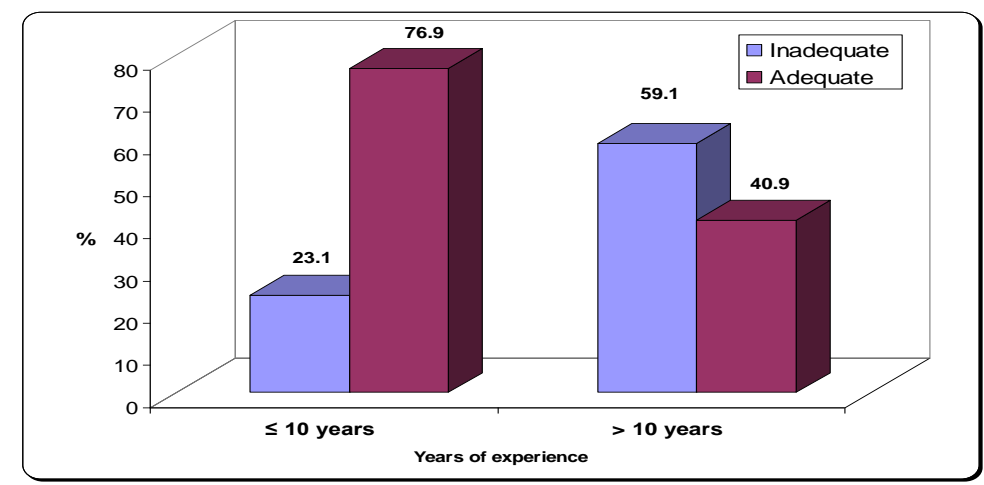

Fig. (4): Relation between total level of practices regarding hemodialysis therapy and years of experience.

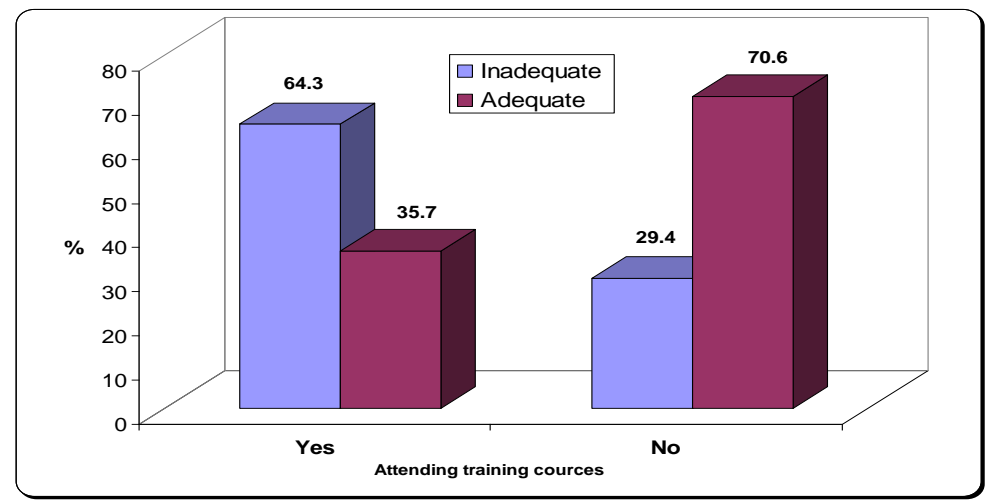

Fig. (5): Relation between total level of practices regarding hemodialysis therapy and attending training courses.

Table (1): Shows personal characteristics of studied nurses. More than three quarters of studied nurses (79.2\%) of AUH were in the age group from 20 years to less than 30 years compare to $(54.2 \%)$ of $\mathrm{MOH}$ nurses with mean \pm SD $30.27 \pm 5.13$. More than three quarters of studied nurses (79.2\%) of $\mathrm{MOH}$ had nursing diplom while $(75.0 \%)$ of AUH nurses had nursing diplom .

According to years of experience it was noticed that more than half $(54.2 \%)$ of studied nurses of AUH had experience from 5 years to less than 10 years compare to(29.2\%) of $\mathrm{MOH}$ nurses. As regarding attending training courses more than half of the studied nurses $(58.3 \%)$ of $\mathrm{MOH}$ had training courses while no nurses of AUH $(0.0 \%)$ attending training courses and according to duration of these courses the majority of studied nurses $(92.9 \%)$ of $\mathrm{MOH}$ had training courses for more than 6 days and most of courses was held out of hospital (78.6\%).

Table (2): Represents Level of nurses' knowledge about renal failure and hemodialysis. It was noticed that the majority of studied nurses of AUH (87.5\%) had good knowledge while $(79.2 \%)$ of $\mathrm{MOH}$ nurses had good knowledge and $(0.0 \%)$ of AUH and $\mathrm{MOH}$ nurses had poor knowledge.
Table (3): Indicates total level of nurses' practices about care of children undergoing hemodialysis. More than three quarters $(79.2 \%)$ of studied nurses of AUH had adequate level of practice compare to $(41.7 \%)$ of $\mathrm{MOH}$ nurses. While $(20.8 \%)$ of $\mathrm{AUH}$ nurses had inadequate level of practice compare to $(58.3 \%)$ of $\mathrm{MOH}$ nurses.

Fig. (1): Shows relation between total level of nurses knowledge regarding renal failure, hemodialysis therapy and their age. There is statistical significance difference between age and level of knowledge for studied nurses $\mathrm{p}$ value $(0.044)$.

Fig. (2): Indicates relation between total level of nurses knowledge regarding renal failure, hemodialysis therapy and years of experience. There is statistical significance difference between years of experience and level of knowledge $p$ value (0.005).

Fig. (3): Shows relation between total level of practices regarding hemodialysis therapy and age of the studied nurses. There is statistical significance difference between age of studied nurses and level of practice with $\mathrm{p}$ value $(0.001)$.

Fig. (4): Represents relation between total level of practices regarding hemodialysis therapy and years of experience. There is statistical significance difference 
between years of experience and level of practice in which $\mathrm{p}$ value $(0.011)$.

Fig. (5): Shows relation between total level of practices regarding hemodialysis therapy and attending training courses. There is statistical significance difference between having training courses and level of practice in which $p$ value (0.025).

\section{Discussion}

Based on the results of the present study, the majority of the nurses their ages ranged from 20- to less than 30 years, married, female, and have diploma of nursing and this agree with Hassan, 2010 stated that the majority of the nurses their ages ranged from 2030 years, married, female, and have diploma of nursing. This study agree with Gadalla, 1998 which stated that the majority of the nurses their ages ranged from 20- to less than 30 years, married, female

As regard of years of experience it was noticed that more than half $(54.2 \%)$ of AUH nurses had experience from 5 years to less than 10 years.while (25.0\%) of $\mathrm{MOH}$ nurses had experience less than 5 years. This study agree with Hassan, 2010 stated that the majority of them their experiences range from 5 to less than 10 years. The present study disagree with Gadalla, 1998 stated that the majority of nurses had experience rang from 1 to less than 5 years.

As regard attending training courses it was found that the majority of studied nurses did not attend training course related to dialysis. This may be attributed to their hospital focus on courses related to infection control rather than courses related to dialysis. This result agree with Hassan, 2010 in which the majority of them have no in-service training courses related to dialysis .

Regarding nurses' knowledge about renal failure and hemodialysis, it was noticed that the majority of nurses $(83.3 \%)$ had high score of knowledge related to care of child undergoing hemodialysis. This may be explained by the most of studied nurses had many years of experience. These agree with Ali, 2011 who stated that the studied nurses were better in their total percent score of knowledge.

On the contrary, results of the present study were inconsistent with Hassan, 2010 who demonstrated that Data collected before the designed nursing protocol implementation (pre-test) showed unsatisfactory level of knowledge about renal failure, hemodialysis and care of patients undergoing hemodialysis.

The present study showed a significant difference between nurse's knowledge scores and age of studied nurses p value (0.044). Nurses' age might be a factor as it plays a vital role in improving the nurse's knowledge. This may be interpreted by the studied nurses spent much time caring for these children. This result are in agreement with Said, 2004 who stated that higher rates of nurses who had satisfactory level existed among the older age of nurses. In contrast Ali, 2011 stated that the nurses who aged less than 20 years had excellent score of knowledge. The present study showed a statistical significant difference between nurse's knowledge scores with their duration of experience and this agree with Hassan, 2010 who stated that there is statistical significant difference between nurse's knowledge scores with their duration of experience.

These results are in disagreement with those of Gadalla, 1998 and Azer, 2005 who reported that total scores of nurses' knowledge in relationship to their years of nurses experience showed that the newly nurses had corrected knowledge more than the old nurses.

The present study revealed that there is no statistical significance difference between having training course and level of knowledge and this agree with Soliman, 2007 who stated that there was no statistical significant difference between nurses who received training program and those who did not receive training program. This disagrees with Ali, 2011 who stated that the nurses who had attending training program had excellent score $(28.8 \%)$ and very good score of their knowledge $(22.5 \%)$.

The present study revealed that generally, nurses had high score in their practice related to care of children undergoing hemodialysis. This may be related to more experience in caring for these children and the availability of resources in the unit. This agree with Hassan, 2010 who stated that the majority of nurses had adequate practice in pre implementing of designed nursing protocol. In contrast Shimokura, et al, 2006 and Buargud, 2005 in their study found that nurse's knowledge was very poor related to all areas of performance.

The result in the present study showed that there is statistical significance difference between age of studied nurses and level of practice with $\mathrm{p}$ value (0.001).This may be related to older nurses depend on younger nurses in work and they prefer to play administrative role only. This agree with Ali, 2011 who stated that the young aged nurses, who is more active, initiative, has good physical fitness and creative in achieving the nursing performance. In contrast Said, 2004 who found that higher rates of nurses who had satisfactory level of performance existed among the older age of nurses.

The results in the present study showed that there is no statistical significant difference between nurses' knowledge and their practice. This may be attributed 
to insufficient courses related to hemodialysis included in their undergraduate curriculum of nursing education and also there is no available Arabic source for updating and continuing their education. This result agree with Hassan, 2010 who stated that there is no significant difference between nurses' knowledge and their practice observed during the pre-test.

The results in the present study showed that there is statistical significant difference between nurses' knowledge scores, and practice with age and this disagree with Hassan, 2010 who stated that there is no significant difference between nurses' knowledge scores, and practice with age.

Also the results showed that there is statistical significant difference between the scores of nurses' practice and their years of experience and this agree with Azer, 2005 who reported that new nurses had a score of performance higher than old nurses. Also this agree with Gamal, 2005 who found the youngest nurses with less than one year experience had better practice than older nurses with more experience.

\section{Conclusion}

Based on the results of the present study, it can be concluded that:

The most of AUH nurses had higher score of knowledge related to renal failure and hemodialysis than the ministry of health hospitals. Nurse's practice regarding hemodialysis children in nephrology unit at Assiut university children hospital is higher than that of nurses working at the Ministry of Heath Hospitals.

\section{Recommendation:}

Based on the results of the present study, the following can be recommended:

1. Continuous nursing educational and in-service training programs on dialysis unit should be well organized within Assiut University children Hospital and equipped with the necessary educational facilities and materials necessary to upgrade the knowledge and skills of practicing nurses, which will be reflected on better outcome and service for the patients.

2. The multidisciplinary approach must be used in caring for children requiring long term dialysis. Available resources should include the child, the child's family, the nurse, and other dialysis staff, renal dietitian, the nephrologists, the social worker and psychologist

3. Periodic monitoring of nurses knowledge and practice to evaluate the level of nurses.
4. The kidney dialysis unit should have a written policy about the standardized nursing care that should be delivered to every patient in the unit.

\section{References:}

1. Ali As, (2011): Knowledge and performance of health team about infection control in the neonatal intensive care units at Assiut and El Minia university hospitals. Unpublished doctoral thesis. Faculty of Nursing, Pediatric nursing department, Assiut university

2. Azer S, (2005): A study of factors affecting nurses' performance. Thesis, B.Sc. Nursing, Faculty of nursing, Adult Nursing department, Assiut University.

3. Barsoum R, (2003): Nephrology in Egypt: A forecast of the Upcoming Century. Available at: http://www.health.egnet.net/health/esn/forecast.h tml.

4. Buargub MA, (2005): The general application of standard health precautions in Tropial ;hemodialysis units. Saudi Journal of kidney Diseases Transplantation;16(2):201-5.

5. Datta P, (2009): Disorders of kidney and urinary tract, Pediatric Nursing, chapter ${ }^{19}, 2^{\text {nd }}$ ed. Jaypee Brothers Medical Publishers (p) LTD, Pp 367372.

6. Gadalla M, (1998): A study of assessment of nurses' performance and knowledge at Assiut hospitals. Thesis, B.Sc. Nursing, Faculty of nursing, Pediatric nursing department, Assiut University.

7. Gamal LM, (2005): Establishing standards for prevention of nosocominal infection in the recovery rooms and surgical ward at Elminia university hospital. Unpublished doctoral thesis. Faculty of Nursing, Assiut university.

1. Hassan GA, (2010): Effect of designed nursing protocol on nurses knowledge and practice regarding hemodialysis patients at Assiut hospitals. Unpublished master Thesis. Faculty of nursing, Adult nursing department, Assiut University.

10. Hockenberry MJ, Wilson D and Winkelstein ML, (2007): Wong's Nursing Care of Infants and Children, Chapter 30 the child with renal dysfunction, $8^{\text {Th }}$ ed. Elsevier Mosby, Pp 122866.

11. Kraut JA, (2009): Chronic renal failure. In: Bope Conn's Current Therapy 2010. Philadelphia: Saunders Elsevier.

12. Kyle T, (2008): Essentials of pediatric Nursing. chapter22, Nursing care of the child with a genitourinary disorders, Lippincott Williams and Wilkins. Pp 743-749. 
13. Perl J, (2009): Home hemodialysis, daily hemodialysis and nocturnal hemodialysis: Core curriculum2009. American Journal of Kidney Diseases.

14. Said HA, (2004): Knowledge and Practices of Nurses regarding Hand washing and Use of Gloves in Patient's Wards in Alexandria Hospitals. Unpublished Master Thesis. High Institute of Public Health University of Alexandria.

15. Seppa and Nathan, (2011): "Bioengineering Better Blood Vessels". Science News.

16. Shimokura G ,Weber D J ,Miller W C, Wurlzel $H$ and Alter M J, (2006): Factors associated with personal protection equipment use and hand hygiene among hemodialysis staff. Am.J.Infect.control;34(3) Pp.100-7.

17. Soliman SM, (2007): Assessment of nurse's knowledge and attitude toward infection standard precaution in primary health care setting. Bulletin of High Institute of public health; 37(1) Pp.189-296.

18. Wolfson AB, (2009): Renal Failure. In: Marx JA, Rosen's Emergency Medicine: Concepts and Clinical Practice. $7^{\text {th }}$ ed. Philadelphia, Mosby. 